\title{
Kinetics of channel formation in bilayer lipid membranes (BLMs) and tethered BLMs: monazomycin and melittin
}

\section{Lucia Becucci and Rolando Guidelli}

Department of Chemistry, Florence University, Via della Lastruccia 3, 50019 Sesto

Fiorentino (Firenze), Italy

\section{Supporting Information}

In general, in a process of nucleation and growth of clusters from monomers, the ratio $S_{x}$ of the "hypothetical" area covered by the growing clusters to that, $\theta_{0}$, initially covered by monomers, if the overlapping of the growing clusters could be ignored, is called the "extended area". This is expressed by the general relationship ${ }^{2,46}$ :

$S_{x}=\int_{0}^{t} A(y, t) v_{N}(y) d y$

where $A(y, t)$ is the area of the cluster nucleated at time $y$ and observed at time $t$. Ascribing for simplicity a circular shape for the growing clusters, the area $A(y, t)$ of a cluster of radius $R$ is given by:

$$
A(y, t)=\pi R(y, t)^{2}=\pi\left[\int_{y}^{t} v_{R}(z) d z\right]^{2}
$$

Therefore, $S_{x}$ takes the form:

$$
S_{x}=\pi \int_{0}^{t}\left[\int_{y}^{t} v_{R}(z) d z\right]^{2} v_{N}(y) d y
$$

The ratio $S$ of the area actually covered by the growing clusters to that initially covered by monomers is related to the corresponding hypothetical "extended" area $S_{x}$, which neglects cluster overlapping, by Avrami's differential equation ${ }^{28-30}$ : 
$d S / d t=(1-S) d S_{x} / d t$

Let us first consider the process of disruption of horizontal clusters and let $\theta_{0}$ denote the initial fraction of the whole electrode surface covered by them. The fraction of $\theta_{0}$ that at a given time $t$ is converted into horizontal monomers will be denoted by $S_{\mathrm{h}}$. Therefore, the fraction of the electrode surface covered by horizontal clusters at a given time $t$ is given by $\theta_{0}\left(1-S_{\mathrm{h}}\right)$. The disruption of horizontal clusters will be treated as a nucleation and growth of "holes" within the surface area covered by the clusters. Let $S_{h, x}$ denote the "extended" area covered by the holes (i.e., the random horizontal monomers resulting from the progressive disruption of horizontal clusters) over that initially covered by the horizontal clusters, upon neglecting overlapping of holes. In view of the general Eq. A3, $S_{h, x}$ is given by:

$S_{h, x}=\pi \int_{0}^{t}\left[\int_{y}^{t} v_{h, R}(z) d z\right]^{2} v_{h, N}(y) d y$

where $v_{h, N}$ and $v_{h, R}$ denote the rates of nucleation and of radial growth of the holes. Differentiation of $S_{h, x}$ with respect to time under the assumption that $v_{h, R}$ is timeindependent yields:

$d S_{h, x} / d t=2 \pi v_{h, R}^{2} u_{h}(t) \quad$ with $: \quad u_{h}(t) \equiv \int_{0}^{t} v_{h, N}(y)(t-y) d y$

Differentiating $u_{h}(t)$ yields:

$d u_{h} / d t=q_{h} \quad$ with $: \quad q_{h} \equiv \int_{0}^{t} v_{h, N}(y) d y$

Finally, upon differentiating $q_{h}$ we get:

$d q_{h} / d t=v_{h, N}(t)=k_{h, N} \theta_{0}\left(1-S_{h}\right)$ 
The relation between $S_{h, x}$ and $S_{h}$ is expressed by the corresponding Avrami's equation (see Eq. A4):

$d S_{h} / d t=\left(1-S_{h}\right) d S_{h, x} / d t$

As sufficiently negative transmembrane potentials, the disruption of clusters is driven by the electric field, which causes the incorporation the horizontal monomers into the lipid bilayer, thus converting them into vertical monomers. Let $p$ denote the potentialdependent ratio of the number of vertical monomers to the number of all monomers (both horizontal and vertical), namely the probability of the monomers being incorporated in the lipid bilayer. Denoting by $S$ the ratio of the area covered by the channel-forming vertical clusters to that covered by both vertical monomers and vertical clusters, the fraction of the whole electrode surface covered by vertical monomers is given by $\Theta=S_{\mathrm{h}} \theta_{0} \mathrm{p}(1-S)$. Let $S_{\mathrm{x}}$ denote the "extended" area corresponding to the "actual" ratio $S$. Differentiating $S_{\mathrm{x}}$ with respect to $t$ via the generalized Leibnitz formula, while taking Eqs. 2, $\mathrm{A} 2$ and $\mathrm{A} 3$ into account, we obtain:

$$
d S_{x} / d t=2 \pi k_{R} \Theta(t) u(t) \quad \text { with }: u(t) \equiv \int_{0}^{t} R(t, y) v_{N}(y) d y
$$

Differentiating $u(t)$ with respect to $t$ yields:

$$
d u / d t \equiv k_{R} \Theta(t) q(t) \quad \text { with }: q(t) \equiv \int_{0}^{t} v_{N}(y) d y
$$

Finally, differentiating $q(t)$ with respect to $t$ and taking Eq. 1 into account we obtain:

$$
d q / d t=v_{N}(t)=k_{N} \Theta^{n}
$$


$S_{\mathrm{h}}(t)$ and $S(t)$ are obtained by solving the system of the eight differential Eqs. A4 and A6A12 by the fourth-order Runge-Kutta method, with the initial conditions $\theta_{0}$ and all other variables equal to zero.

The current density $I(\mathrm{t})$ is proportional to the fraction of the electrode surface covered by the channel-forming vertical clusters:

$I(t)=S_{h} \theta_{0} S p$

The calculation of the charge flowing across a Hg-supported lipid bilayer incorporating an ion channel requires the estimate of the contribution from the radial diffusion of the permeating ions in the hydrophilic spacer from the mouth of the ion channel, starting from the instant, $y$, at which it is formed by nucleation and growth. The extended area $S_{\mathrm{d}, \mathrm{x}}$ covered by the "diffusion disk" at a given time $\mathrm{t}$ is expressed by the equation:

$$
S_{d, x}=\pi^{2} \int_{0}^{t} R^{2}(y, t) R_{d}^{2}(y, t) v_{N}(y) d y
$$

where $R_{\mathrm{d}}$ is the radius of the diffusion disk. Under the assumption that time derivative of $R_{d}^{2}$ is constant and equal to $k_{\mathrm{d}, \mathrm{R}}$, differentiation of $S_{\mathrm{d}, \mathrm{x}}$ via the generalized Leibnitz formula, while taking Eq. 2 into account, yields:

$$
d S_{d, x} / d t=2 \pi^{2} k_{R} \Theta(t) m(t)+\pi^{2} k_{d, R} w(t)
$$

with:

$$
m(t)=\int_{0}^{t} R(t, y)\left[R_{d}(t, y)\right]^{2} v_{N}(y) d y \quad ; \quad w(t)=\int_{0}^{t}[R(t, y)]^{2} v_{N}(y) d y
$$

A further differentiation with respect to time yields:

$$
d m / d t=k_{d, R} u(t)+k_{R} \Theta(t) z(t)
$$

and: 
$d w / d t=2 k_{R} \Theta(t) u(t)$

with $u(t)$ given by Eq. A10 and $z(t)$ given by:

$z(t)=\int_{0}^{t}\left[R_{d}(y, t)\right]^{2} v_{N}(y) d y$

Differentiating $z(t)$ yields:

$d z / d t=k_{d, R} q(t)$

where $q(t)$ is given by Eq. A11. $S_{\mathrm{d}}$ is related to $S_{\mathrm{d}, \mathrm{x}}$ by the corresponding Avrami's differential equation (see Eq. A4):

$d S_{d} / d t=\left(1-S_{d}\right) d S_{d, x} / d t$

The normalized charge $Q(t)$ is given by the fraction of the whole electrode surface area covered by the channel-forming clusters, $S_{\mathrm{h}} \theta_{0} \mathrm{p} S$, plus the fraction that is progressively covered by the ions diffusing from the mouth of the growing channels:

$Q(t)=S_{h} \theta_{0} p S+\left(1-S_{h} \theta_{0} p S\right) S_{d}$

$Q(t)$ is obtained by solving the system of the thirteen differential equations A4, A6-A12, A15, A17, A18, A20 and A21 by the fourth-order Runge-Kutta method. 